\title{
O conhecimento dos estudantes de enfermagem sobre a hipodermóclise no idoso
}

\author{
The knowledge of nursing students about hypodermochclise in the elderly
}

\author{
Samilla Gonçalves Ferreira de Menezes', Maria Olívia Sobral Fraga de Medeiros² \\ 'Autora para correspondência. Escola Bahiana de Medicina e Saúde Pública, Salvador, Bahia, Brasil. \\ ORCID: 0000-0002-8401-5183. samillaferreira@hotmail.com \\ ${ }^{2}$ Escola Bahiana de Medicina e Saúde Pública, Salvador, Bahia, Brasil. ORCID: 0000-0001-6811-378X. mariamedeiros@bahiana.edu.br
}

RESUMO | OBJETIVO: avaliar o conhecimento dos estudantes de Enfermagem sobre o uso da hipodermóclise no idoso. MÉTODO: Pesquisa com caráter quantitativo-descritivo, realizada com 119 estudantes de enfermagem que já cursaram ou estão cursando a disciplina com abordagem em Técnica de Administração I e II, mediante entrevista semiestruturada autoaplicável no contexto de uma universidade privada localizada na cidade de Salvador-BA, nos meses de setembro e outubro de 2016. RESULTADOS: 71 participantes responderam não conhecer a técnica, sendo que maioria desses estudantes não vieram transferidos de outra instituição e não possuem nenhuma formação técnica.

DESCRITORES: Hipodermóclise. Idoso. Administração cutânea. Estudantes de enfermagem.

\begin{abstract}
OBJECTIVE: to evaluate the knowledge of nursing students about the use of hypodermoclisis in the elderly. METHOD: This is a quantitative-descriptive research carried out with 119 nursing students who have already studied or are attending the course with a Management Technique I and II approach, using a semistructured self-administered interview in the context of a private university located in the city of Salvador, Bahia, in the months of September and October 2016. RESULTS: 71 respondents did not know the technique, most of whom were not transferred from another institution and did not have any technical training.
\end{abstract}

KEYWORDS: Hypodermoclysis. Elderly. Cutaneous management. Nursing students. 


\section{Introdução}

O processo de envelhecimento populacional é um fenômeno mundial. No Brasil esse crescimento acontece de forma acelerada, no qual em projeções mais tradicionalistas mostram que seremos o sexto país em maior número de idoso, com um número maior que 30 milhões de pessoas ${ }^{1,2}$.

Com o envelhecimento da população, tem-se observado o aumento de morbidades, fragilidades na atenção primária (promoção e prevenção à saúde) e consequentemente altas taxas de internação hospitalar (seja para tratamentos clínicos, procedimentos diagnósticos ou terapêuticos). As alterações anatomofisiológicas do envelhecimento tegumentar e vascular associados a presença de fatores senis que desencadeiam em importantes limitações que impedem a manutenção de acessos venosos perifé$\operatorname{ricos}^{3,4}$.

É nesse contexto, que a prática da hipodermóclise, pode ser uma via de administração parenteral alternativa e eficaz. Desde 1903 a técnica era utilizada em hospitais para hidratação de pacientes e costumava ser comum, particularmente na medicina geriátrica. Contudo, em meados de 1950 a concernente prática entrou em desuso, pois esteve associado a eventos iatrogênicos que ocorreram por sobrecarga hídrica e ao choque circulatório após a infusão de grandes volumes pela via subcutânea. Ao fim dos anos 60, com o advento dos Cuidados Paliativos na Inglaterra, a hipodermóclise voltou a ser considerada como uma via segura de administração e então a partir da década de 80 que passou a integrar a paliação e principalmente no cuidado aos idosos. No Brasil, essa técnica vem ganhando, aos poucos, seu espaço, ou pelo menos retorno aos pontos de discussão e estudo ${ }^{3,4,5}$.

A hipodermóclise ou terapia subcutânea é a infusão de fluidos isotônicos e/ou medicamentos por via subcutânea. Tem como finalidade a reposição hidroeletrolítica e/ou terapia medicamentosa, sendo recomendada na prática clínica e vem sendo utilizada principalmente em idosos, pacientes oncológicos e em cuidados paliativos. Entretanto ainda é pouco utilizada no Brasil'.

O conhecimento desta técnica permite ao enfermeiro maior possibilidade de atuação quanto ao con- trole de sintomas, sobretudo, em pacientes idosos e em indivíduos em cuidados paliativos, contribuindo para uma melhora na qualidade de vida e proporcionando maior conforto e segurança para pacientes, familiares e equipe de trabalho. Portanto, desenvolver e incentivar novos estudos favorecem a consolidação da terapêutica e a ampliação da discussão sobre a temática nos ambientes institucionais de saúde, escolas e na docência ${ }^{3,4}$.

Dentro deste contexto, esse trabalho objetiva avaliar o conhecimento dos estudantes de Enfermagem sobre o uso da hipodermóclise no idoso.

\section{Método}

Trata-se de uma pesquisa descritiva, com abordagem quantitativa cuja principal finalidade é o delineamento e análise das características de fatos ou fenômenos $s^{6,7}$. Foi realizada entre os meses de setembro e outubro de 2016, com 119 alunos de uma instituição de ensino superior privada do município de Salvador-Ba.

A população do estudo foi composta por discentes do $3^{\circ}, 4^{\circ}, 5^{\circ} 6^{\circ}, 7^{\circ}$ e $9^{\circ}$ semestres do curso de enfermagem. Os critérios de inclusão foram: ser discente de enfermagem e ter cursado ou estar cursando disciplinas que abordam técnica de administração medicamentosa. A partir da matriz curricular foi eleita as disciplinas Técnica de Administração I e/ ou Técnica de administração II como suporte teórico para a prática assistencial.

Os critérios de exclusão foram: discentes do $8^{\circ}$ semestre, que apesar de já terem cursado as disciplinas de apoio teórico, tiveram acesso a essa pesquisa, ainda enquanto projeto, como participação avaliativa da disciplina obrigatória Metodologia de Pesquisa Cientifica. Além destes, foram excluídos alunos afastados por motivos médicos (atestados médicos e licença maternidade).

Para a coleta de dados foi utilizado um questionário semiestruturado e autoaplicável, elaborado pelas pesquisadoras, contendo um interrogatório social (identificação, data de nascimento, semestre em curso na graduação, se já havia cursado a disciplina, 
ano de ingresso e se esse foi por transferência interinstitucional, formação profissional anterior) além de cinco questões discursivas acerca da temática. Após a realização de toda a coleta de dados, foi entregue um folder informativo sobre a hipodermóclise, visando contribuir no processo educativo dos estudantes.

As informações coletadas foram armazenadas e tabuladas no programa software Excel Microsoft versão 2010. E os dados foram analisados por meio de estatística descritiva simples.

A pesquisa obteve aprovação pelo Comitê de Ética e Pesquisa da instituição sob CAAE $n^{\circ}$ 565201716.4.0000.55544, conforme indicação do Conselho Nacional de Ética e Pesquisa (CONEP), observando-se ainda os aspectos éticos da Resolução 466/2012 do Conselho Nacional de Saúde, o qual dispõe sobre a ética na pesquisa envolvendo seres humanos. Antes da coleta de dados, os estudantes foram esclarecidos sobre os objetivos da pesquisa e manifestaram sua concordância por meio da anuência com a assinatura Termo de Consentimento Livre e Esclarecido.

\section{Resultados}

Foram 119 questionários respondidos pelos estudantes de enfermagem dos quais 110 eram do sexo feminino e a faixa etária prevalente foi de 18 a 27 anos, sendo a idade máxima encontrada de 47 anos. Desses, 30 estudantes são originários de transferência de outra instituição de ensino superior, 8 já haviam cursado a disciplina com abordagem para procedimentos/técnicas em enfermagem. Quanto à formação profissional 20 estudantes já tinham formação técnica e 11 declararam exercer alguma atividade trabalhista, tais como: técnico em enfermagem, técnico em instrumentação cirúrgica, técnico em segurança do trabalho e técnico em manutenção automobilística.

No que se refere ao conhecimento dos discentes de enfermagem sobre a técnica de hipodermóclise, apenas $48(40,34 \%)$ afirmaram conhecê-la, conforme fluxograma 1.

Fluxograma 1. Perfil dos discentes de enfermagem transferidos de outra intuição que disseram conhecer a hipodermóclise

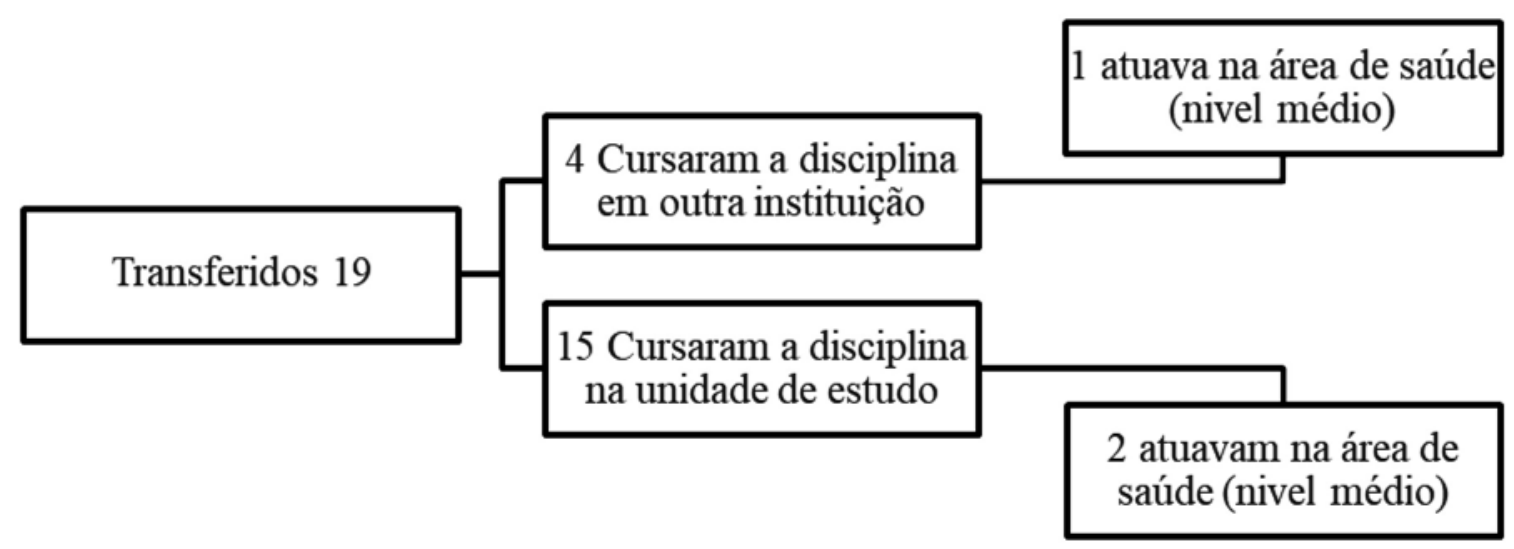

Fonte: As autoras (2018).

Dentre os discentes que conhecem a técnica de hipodermóclise, 19 alunos eram provenientes de outra instituição de ensino superior (IES) por transferência externa, 29 eram originários da própria instituição do estudo, conforme fluxograma 2. 


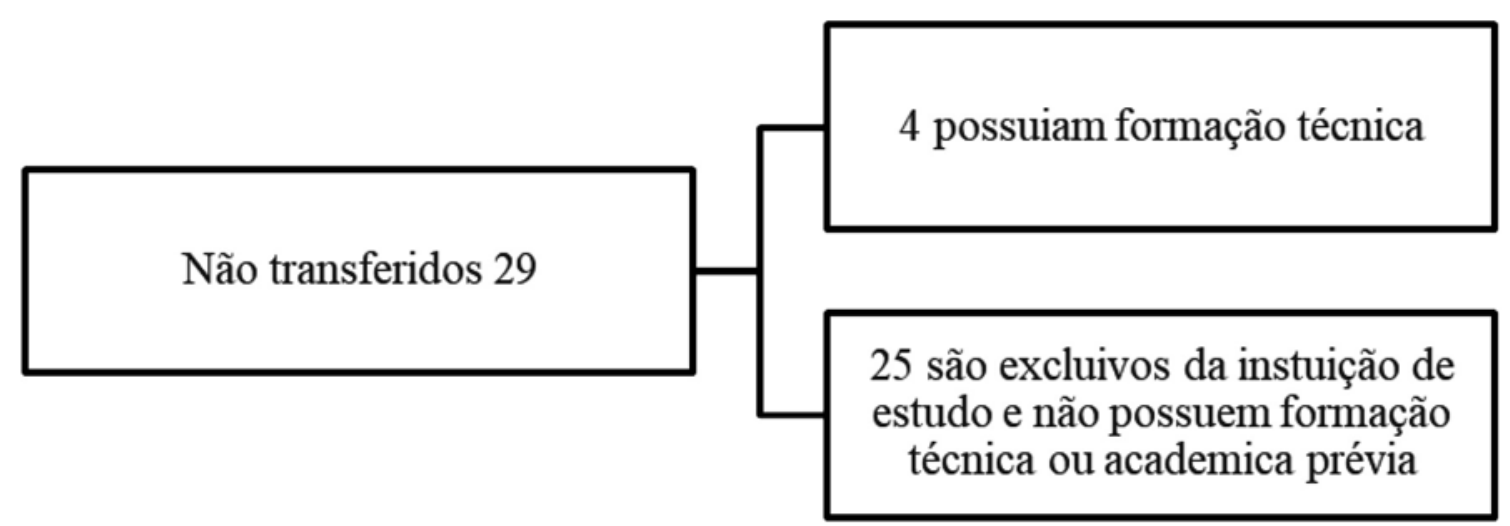

Fonte: As autoras (2018).

\section{Discussão}

É possível inferir que a instituição de estudo tem abordado a temática, o que é corroborado pelo percentil de alunos que conhecem o tema. Todavia é importante ressaltar que existem indivíduos participantes que já atuavam na área de saúde, aumentando possivelmente $o$ índice de acertos. Um estudo com metodologia semelhante, mas com graduados, apontou que $71 \%$ dos enfermeiros desconheciam completamente a técnica, o que aponta para a incipiência do conhecimento ${ }^{8}$.

Os discentes referiram que 0 momento de aproximação com a temática ocorreu a partir do $2^{\circ}$ ano de faculdade e elegeram os $3^{\circ}, 4^{\circ}, 5^{\circ}$ e $7^{\circ}$ semestres como os que mais enfatizavam 0 tema dentre o conteúdo programático das disciplinas. Tal concordância coaduna as ementas curriculares das disciplinas ofertadas, que definem os cuidados de enfermagem de maior complexidade técnica, como os que exigem conhecimentos de base cientifica e capacidade de tomar decisões imediatas, dos quais a administração medicamentosa faz parte?

Foi possível elencar que a matéria de Técnicas de administração II realizada no $4^{\circ}$ semestre foi reconhecida pela maioria dos discentes como a que mais favoreceu conhecimento sobre a hipodermóclise (Gráfico 1).

Gráfico 1. Momento que os estudantes referiram aproximação com a hipodermóclise

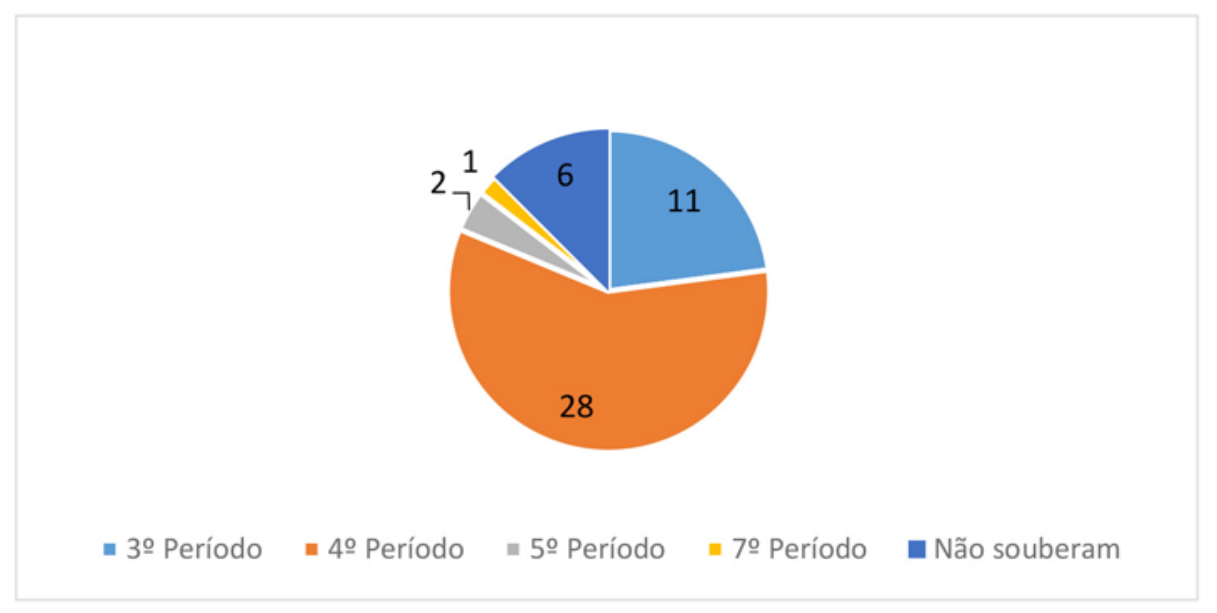

Fonte: As autoras (2018). 
Dezenove estudantes apontaram como indicação para uso da hipodermóclise: "pacientes não queimados", "com dificuldade de acesso", "com necessidade de tratamento", "em cuidados paliativos", "com dor crônica", "pessoas com fragilidade capilar", "pacientes em tratamento oncológico" e "pacientes idosos". O que é validado pela literatura que define a hipodermóclise como uma via de escolha para os pacientes que necessitam de suporte clínico para reposição de fluidos, administração de medicamentos e eletrólitos, e que tenham dificuldades de acesso vascular periférico associado a fragilidade capilar, a sucessivas e mal-sucedidas tentativas de punção venosa periférica, presença de várias flebites, sinais flogísticos e histórico de trombose venosa $a^{3,4,10}$.

A hipodermóclise agrega várias vantagens como: baixo custo, baixos índices de infecção, indicação para cuidado domiciliar, facilidade de obter novos sítios de inserção, possibilidade de ser interrompida e reiniciada, favorecimento da funcionalidade do paciente, boa aceitação por parte dos familiares, dispensa a imobilização do membro. É sobretudo vantajosa por diminuir as complicações vasculares sistêmicas, além de permitir a liberação prolongada e a disponibilidade sérica sustentada da medicação, proporcionando alívio de sintomas por mais tempo ${ }^{11}$. Apesar dos benefícios, ainda é possível identificar lacunas teóricas, 71 estudantes afirmaram desconhecer a hipodermóclise.

Por ser considerada uma via segura e confortável para o paciente, e reduzir efeitos indesejáveis na pele, alguns cuidados de enfermagem são indispensáveis, tais como: lavar as mãos antes de manusear o cateter; fazer assepsia da via de acesso sempre que abrir o sistema, orientar o paciente, familiares e equipe sobre a possibilidade de discreta hiperemia e edema no local de inserção do cateter logo após a punção e proteger a punção com plástico durante o banho para manter a área seca $^{12}$. Salienta-se que quando a técnica correta de punção é realizada e os padrões de diluição/ velocidade de infusão dos medicamentos são respeitadas, são raras as complicações.

A terapia subcutânea, hipodemóclise, ressurge como via de hidratação com potenciais vantagens para população idosa, embora pouco se saiba sobre sua aplicação em ambiente hospitalar. A eficá- cia da reidratação subcutânea em pacientes idosos hospitalizados com desidratação leve-moderada não é inferior à obtida por via intravenosa e apresentar vantagens adicionais ${ }^{13}$.

Os cuidados a serem demandados ao utilizar-se desse método foram empregados como ferramenta educativa num estudo de intervenção com enfermeiros que revelou tais práticas como medida crucial para propiciar uma redução significativa de celulite após punção na população geriátrica. A educação em enfermagem foi apontada como vital, pois identificou e corrigiu os déficits de conhecimento na prática $^{14}$.

Dentre os efeitos complicadores é possível elencar: edema local, dor, calor, endurecimento, necrose, eritema ao redor do sítio de inserção do cateter (esperado nas primeiras 4 horas), extravasamento pelo óstio e a presença de infecção. Há um baixo risco de disseminação sistêmica, pois não ocorre acesso ao espaço vascular, e mesmo que essa infecção ocorra, ela possui uma tendência a se limitar ao local da inserção, podendo ser facilmente controlada ${ }^{15,16}$.

Apenas três estudantes apontaram efeitos complicadores associados ao volume a ser infundido e aos aspectos fisiológicos do envelhecimento como: "via em que não pode ser administrada muitos volumes", "diminuição da absorção que pode causar edema subcutâneo", "turgor diminuído que dificulta a punção".

A importância de se abordar temas como esses durante a graduação é condição indispensável para uma assistência de enfermagem mais coesa. O enfermeiro é responsável pela terapêutica medicamentosa administrada aos pacientes (seja por supervisão ou preparo direto) e cabe a esse profissional evitar ou minimizar eventos adversos/ iatrogênicos. A administração de medicamentos, a capacidade de avaliar, observar e registrar os achados encontrados durante a punção e após, constituem importantes responsabilidades e deveres do profissional ${ }^{17}$.

O uso adequado da hipodermóclise proporciona conforto e alívio dos sintomas para o paciente e sua família, além de possibilitar segurança para a equipe de saúde envolvida no processo de cuidado ${ }^{18}$. Conforto e alivio dos sintomas foram apontados por 
16 estudantes como benefícios diretos. Além da "redução dos riscos de infecção", "oferta gradual de medicação e menor reação adversa", "via alternativa/ falta de acesso periférico", "via menos agressiva e menos invasiva", "confortável ao paciente e melhor possibilidade de acesso", "maior perfusão com duração estendida" e "facilidade de absorção de medicamentos".

Esta pesquisa proporcionou o estímulo pela busca de conhecimento acerca da hipodermóclise por parte dos discentes de enfermagem, apontando possíveis lacunas no arcabouço teórico. Todavia, apresenta como limitação a metodologia adotada que não permitiu explorar as experiências dos estudantes, e o não acesso ao conteúdo programático elaborado pelos docentes da instituição. Numa tentativa de sensibilizar sobre a temática foi fornecido um panfleto educativo.

\section{Considerações finais}

Apesar da importância do uso da hipodermóclise para a prática assistencial, observou-se que apenas $44 \%$ estudantes do último semestre do curso conheciam a terminologia e a técnica. Tal realidade aponta para a possibilidade de retroalimentar uma cadeia de não utilização da técnica na prática assistencial, seja por vulnerabilidades no conhecimento científico, ou seja, falta de protocolos e incentivos institucionais.

\section{Contribuição dos autores}

Menezes SGF contribuiu na concepção do projeto, coleta, análise e interpretação dos dados, redação do artigo e aprovação da versão final a ser publicada. Medeiros MOSF contribuiu na concepção do projeto, análise e interpretação dos dados e redação do artigo, revisão crítica relevante do conteúdo e aprovação da versão final a ser publicada.

\section{Conflitos de interesses}

Nenhum conflito financeiro, legal ou político envolvendo terceiros (governo, empresas e fundações privadas, etc.) foi declarado para nenhum aspecto do trabalho submetido (incluindo mas não limitandose a subvenções e financiamentos, conselho consultivo, desenho de estudo, preparação de manuscrito, análise estatística, etc).

\section{Referências}

1. Santana RCB. Cuidado de enfermagem à pessoa idosa hospitalizada com necessidade de acesso venoso periférico [monografia]. Salvador: Universidade Federal da BahiaUFBA; 2014.

2. Veras R. Envelhecimento populacional contemporâneo: demandas, desafios e inovações. Rev Saúde Pública. 2009;43(3):548-54. doi: 10.1590/S0034$\underline{89102009005000025}$

3. Bruno VG. Hipodermóclise: Revisão de literatura para auxiliar a pratica clínica. Hospital Israelita Albert Einstein. $2013 ; 13(1): 122-8$.

4. D'Aquino MO, Souza RM. Hipodermóclise ou Via Subcutânea. Revista do Hospital Universitário Pedro Ernesto. 2012;11:89-93.

5. Gomes NS, Silva AMB, Zago LB, Silva ECL, Barichello E. Conhecimentos e práticas da enfermagem na administração de fluidos por via subcutânea. Rev Bras Enferm. 2017;70(5):1 155-64. doi: 10.1590/0034-7167-2016-0424

6. Zironde ES, Marzenini NL, Soler VM. Hipodermóclise: redescoberta da via subcutânea no tratamento de indivíduos vulneráveis. Revista Cuidarte Enfermagem. 2014;8(1):55-61.

7. Marconi MA, Lakatos EM. Fundamentos da metodologia científica. 7.ed. São Paulo: Editora Atlas; 2010.

8. Takaki CYI, Klein GFS. Hipodermóclise: o conhecimento do enfermeiro em unidade de internação. Revista ConScientiae Saúde. 2010;9(3):486-496.

9. Cofen. Resolução do COFEN n 358/2009. Dispõe sobre a Sistematização da Assistência de Enfermagem e a implementação do Processo de Enfermagem em ambientes, públicos ou privados, em que ocorre o cuidado profissional de Enfermagem, e dá outras providências.

10. Justino ET, Tuoto FS, Kalinke LP, Mantovani MF. Hipodermóclise em pacientes oncológicos sob cuidados paliativos. Cogitare Enfermagem. 2013;18(1):84-9. doi: $\underline{10.5380 / \text { ce.v18i1.31307 }}$

11. Santos RCJ. Cuidado de Enfermagem na Terapia Subcutânea/Hipodermóclise [Internet]. 2012 [acesso em 2015 set 5]. Disponível em: http://bvsms.saude.gov.br/bvs/ publicacoes/inca/Rita_Santos_cuidado_enfermagem.pdf

12. Lima DA, organizador. $O$ uso da via subcutânea em geriatria e cuidados paliativos. Rio de Janeiro: Sociedade Brasileira de Geriatria e Gerontologia; 2016.

13. Slesak G, Schnürle JW, Kinzel E, Jakob J, Dietz PK. Comparison of Subcutaneous and Intravenous Rehydration in Geriatric Patients: A Randomized Trial. J Am Geriatr Soc. 2003;51(2):155-60. 
14. Candon HL, Toen JV, Amirov C. Uma intervenção multifacetada para abordar um grupo de casos de celulite associada à hipodermóclise em uma unidade de cuidados continuados complexos geriátricos. Canadian Journal of Infection Control. 2010;25(2):101-106.

15. Braz CL, Pereira RCC, Costa JM. Administração de medicamentos por hipodermóclise: uma revisão da literatura. Rev Bras Farm Hosp Serv Saúde. 2015;6(1):6-12.

16. Pedreira MLG, Hipodermóclise. In: Harada MJCS, Pedreira MLG, organizadores. Terapia intravenosa e infusões. São Caetano do Sul: Yendis; 2011 . p. 475-488.

17. Oliveira FFS, Carvalho ESS. Hipodermóclise: Alternativa para Administração de Drogas e Soluções no Contexto dos Cuidados Paliativos. In: Silva RS, Amaral JB, Malaguetti W. Enfermagem em Cuidados Paliativos: cuidando para uma boa morte. Martinari; 2013. p. 335-349.

18. Serbim AK, Remor CB. Enfermagem em cuidados paliativos. In: Dalacorte RR, Rigo JC, Schneider RH, Scwanke CHA. Cuidados paliativos em Geriatria e Gerontologia. São Paulo: Editora Atheneu; 2012. p. 230-232.

19. Kauark FS, Manhães FC, Medeiros CH. Metodologia da pesquisa: um guia prático. Itabuna: via litterarum editora; 2010.

20. Pontalti $G$, Rodrigues ESA, Firmin F, Fábris $M$, Stein $M R$, Longara VK. Via subcutânea: segunda opção em cuidados paliativos. Revista HCPA. 201 2;32(2):199-207. 